\title{
LA PRENSA PEDAGÓGICA EN LA RECONSTRUCCIÓN DEL PASADO EDUCATIVO: EL CASO DE LA REVISTA ESCUELAS DE ESPAÑA (1929-1936)
}

DOI: http://dx.doi.org/10.1590/2236-3459/78399

\author{
Rosa Ortiz de Santos' \\ 'Universidad de Valladolid (UVA), Segovia, España \\ Luis Torrego Egido" \\ "Universidad de Valladolid (UVA), Segovia, España
}

$\cos 8$

\begin{abstract}
Resumen
Escuelas de España es una de las revistas pedagógicas más representativas de la educación y de las escuelas de la Segunda República en España. La revisión de los estudios internacionales sobre la prensa pedagógica evidencia el escaso tratamiento de la revista por parte de la investigación histórico-educativa. Este artículo pretende contribuir a paliar esa carencia, pues en él se realiza un análisis descriptivo de la publicación, partiendo de su valor como patrimonio pedagógico que permite investigar la educación y la renovación pedagógica realizadas desde 1929 hasta 1936. Se estudian su origen, propósitos y características, la estructura, las secciones y los contenidos, así como a sus protagonistas, destacando a los tres maestros que la fundaron y dirigieron.

Palabras clave: Escuelas de España, prensa pedagógica, renovación pedagógica, patrimonio históricoeducativo, España.
\end{abstract}

\section{A IMPRENSA PEDAGÓGICA NA RECONSTRUÇÃO DO PASSADO EDUCATIVO: O CASO DA REVISTA ESCUELAS DE ESPAÑA (1929-1936)}

\section{Resumo}

Escuelas de España é uma das revistas pedagógicas mais representativas da educação e das escolas da Segunda República da Espanha. A revisão de estudos internacionais sobre a imprensa pedagógica põe em evidência e mostra o escasso tratamento da revista pela pesquisa histórico-educacional. Este artigo pretende contribuir atenuando essa carência, pois nele se realiza uma análise descritiva da publicação, partindo do seu valor como uma herança e patrimônio pedagógico que permite pesquisar la educação e la renovação pedagógica de 1929 a 1936. Se estuda sua origem, propósitos e características, estrutura, seções e conteúdos, bem como seus protagonistas, destacando os três professores que fundaram e dirigiram.

Palavras-chave: Escuelas de España, imprensa pedagógica, renovação pedagógica, patrimônio históricoeducacional, Espanha. 
THE PEDAGOGICAL PRESS IN THE RECONSTRUCTION OF THE EDUCATIONAL PAST: THE CASE OF THE JOURNAL ESCUELAS DE ESPAÑA (1929-1936)

\begin{abstract}
Escuelas de España is one of the most representative pedagogical journals of education and of the schools of the Second Republic in Spain. The review of the international studies on pedagogical press shows the scarce historical and educational research carried out on this journal. This article aims to help fill that gap, as it features a descriptive analysis of that publication. The analysis is based on its value as a pedagogical heritage that allows researching the education and pedagogical renovation from 1929 to 1936. Its origin, purposes and characteristics, structure, sections and contents are studied, as well as its protagonists, especially the three teachers who founded and ran it.

Keywords: Escuelas de España, pedagogical press, pedagogical renovation, historical-educative patrimony, Spain.
\end{abstract}

\title{
LA PRESSE PEDAGOGIQUE DANS LA RECONSTRUCTION DU PASSÉ ÉDUCATIF: LE CAS DU MAGAZINE DES ESCUELAS DE ESPAÑA (1929-1936)
}

\section{Résumé}

Escuelas de España est l'une des revues pédagogiques les plus représentatives de l'éducation et des écoles de la Deuxième République en Espagne. La revue des études internationales sur la presse pédagogique montre le peu de traitement de la revue par la recherche historico-pédagogique. Cet article vise à pallier ce manque, puisqu'il s'agit d'une analyse descriptive de la publication, fondée sur sa valeur en tant qu'héritage pédagogique permettant de reconstituer la mémoire de l'éducation et du renouveau pédagogique de 1929 à 1936. Son origine est étudiée, buts et caractéristiques, structure, sections et contenus, ainsi que ses protagonistes, en soulignant les trois enseignants qui l'ont fondé et dirigé.

Mots-clés: Escuelas de España, presse pédagogique, renouvellement pédagogique, patrimoine historieéducatif, Espagne. 


\section{Introducción}

a prensa "es un instrumento de comunicación social arraigado en la vida cotidiana de los ciudadanos". (HERNÁNDEZ, 2013, p. 15). A lo largo de la historia ha constituido una fuente de intercambio, circulación y transmisión de opiniones, ideas e informaciones, y un medio para la defensa de ideales y derechos y para el establecimiento de relaciones entre las personas. Constituye, por tanto, el propio engranaje social y conforma un elemento de crítica y análisis de los procesos políticos, culturales, educativos (ERMEL, 2017), económicos y sociales de una comunidad en un momento determinado. La prensa es un instrumento con gran significación para el análisis y la interpretación en la investigación histórica y social.

Las cuestiones educativas se recogen de forma especializada en la prensa pedagógica. La ingente cantidad de títulos pedagógicos que se han ido sucediendo a lo largo del tiempo permite estudiar "el desarrollo y la expansión de las ideas educativas, las concepciones pedagógicas vigentes, la evolución de la práctica docente, los problemas profesionales del magisterio" (TIANA, 1988, p. 150), en definitiva, interpretar y conocer la educación y las escuelas del pasado. En consecuencia, la prensa pedagógica se convierte en un valioso instrumento o fuente y objeto de investigación en historia de la educación.

Un importante apunte es el que sostiene Hernández (2013) defendiendo el valor que tiene la prensa pedagógica en sí misma y caracterizándola como patrimonio históricoeducativo del ayer, constituyendo un camino para acceder al estudio de las realizaciones educativas. Existen, además, otros autores de referencia en el ámbito internacional que estudian el valor de la prensa especializada en educación como un medio esencial en la recuperación y reconstrucción de la historia de la educación. Petchak y Moura (2013) indican que los contenidos de las publicaciones pedagógicas evidencian el papel que la prensa tuvo en la formación de opiniones y en la construcción de una identidad ideológica, política, social y cultural determinada. (RODRIGUES, 2010). Caspard-Karydis (2000) y Campbell (2013) consideran que la prensa pedagógica es un fiel reflejo de la vida social imperante en un momento espaciotemporal concreto, pues nos permite conocer "el discurso pedagógico, las prácticas educativas, la cotidianeidad escolar, el grado de sumisión de los profesores a los programas y las instituciones oficiales, la ideología oficial y el cuerpo docente". (BASTOS, 2016, p. 3). Asimismo, contribuye a destapar la fuerza y la riqueza de la renovación pedagógica y de la innovación educativa que, en muchos casos, puede haberse visto encubierta (BASTOS, 2007) o haber sido ignorada. Este es, precisamente, el caso de la revista pedagógica.

\section{La prensa pedagógica en España (1929-1936)}

En el contexto de la dictadura de Miguel Primo de Rivera (1923-1930) hubo un evidente decaimiento en la producción de títulos pedagógicos en el contexto español. No obstante, y aunque en menor proporción, continúan apareciendo nuevas publicaciones; este es el caso de la revista Escuelas de España, que tuvo su inicio en 1929.

Durante la Segunda República Española (1931-1936) el interés y la preocupación por la educación y las escuelas crecieron de forma considerable, siendo una época de cambios sustanciales en la prensa pedagógica española. "El tono del ambiente ya ofrecía caldo suficiente para la proliferación de revistas y empresas pedagógicas". (LOZANO, 1980, 
p. 189). En este sentido, la etapa de la historia en la que se publica Escuelas de España coincide con la que Viñao (1994-1995) denomina "edad de plata' de la cultura española y que, en cuanto a la educación se refiere, son años de renovación y reformas, de apertura al exterior y floración de una serie de revistas". (p. 9). Algunas de las publicaciones pedagógicas que enumeran este autor, Lozano (1980) y Esteban y López (1992) son la Revista de Pedagogía (1922-1936), fundada por Lorenzo Luzuriaga, el Boletín de la Institución Libre de Enseñanza (1877-1936), La Escuela Moderna (1891-1934), la Revista de Escuelas Normales (1923-1936), la Revista de Psicología i Pedagogía (1933-1937), Baixeras (1925-1930), de Félix Martí Alpera, El Magisterio Español (1867-1934), Residencia (1926-1934), además de la revista segoviana Escuelas de España.

Es reseñable que pese a la baja densidad de población y ante un contexto rural, como el que predominaba en Castilla y León, cada una de sus capitales contaba con más de un periódico educativo, lo que evidencia una gran riqueza pedagógica (CHECA, 2002), siendo Segovia una de las provincias españolas en las que más títulos se produjeron durante la última etapa de la dictadura. A juicio de este mismo autor, las publicaciones estaban, en su mayoría, impulsadas por docentes locales y orientadas a atender preferentemente a las necesidades e inquietudes de los docentes de primera enseñanza.

Coincidimos con Checa (2002) cuando señala que "estas publicaciones están pidiendo estudios monográficos, que ya han comenzado a surgir de las más significativas o las más accesibles". (p. 41). Por eso, en este artículo se presenta la revista pedagógica Escuelas de España, partiendo del escaso tratamiento que ha tenido en el ámbito de la investigación histórico-educativa, a diferencia de otras publicaciones pedagógicas de referencia.

\section{El estudio de la prensa pedagógica del primer tercio del siglo XX en España}

La existencia de publicaciones dedicadas al análisis de la prensa pedagógica de las tres primeras décadas del siglo XX muestra el interés que tiene la comunidad científica por conocer el modo de pensar la educación y la pedagogía durante este periodo, pudiendo contribuir a la construcción del patrimonio histórico-educativo de España. Historiadores, periodistas, pedagogos y docentes han estudiado el pasado educativo a través de las revistas pedagógicas.

Uno de los análisis más completos de las publicaciones pedagógicas de España es el de Checa (2002), quien refleja la evolución de este tipo de prensa desde sus inicios hasta la actualidad en las diferentes comunidades autónomas. Algo similar se observa en el trabajo de Esteban y López (1992), quienes realizan un análisis general del periodismo pedagógico desde 1803 hasta 1940 y lo acompañan de un índice de 867 revistas de esta misma índole y periodo temporal. Por su parte, se encuentran investigaciones más localistas como la tesis doctoral de Cachazo (2015), que analiza la prensa pedagógica de Cataluña entre 1820 y 1939.

Se pueden localizar, además, artículos científicos dedicados a la presentación de revistas históricas pedagógicas de referencia. Aritmendi (2009) y Viñao (1994-1995) realizan un análisis general de la Revista de Pedagogía, presentando sus características y el contexto en el que se encuadra. Existen otros autores y autoras que han examinado el tratamiento de contenidos particulares en la obra. Entre ellos, López y Delgado (2014) valoran la presencia de la enseñanza de las ciencias, Ermel (2017) realiza una revisión de 
los artículos relacionados con los edificios y el material escolar y Alfaro y Carpintero (1983) analizan cómo se aborda la psicología en educación dentro de la revista.

Otra publicación que ha sido objeto de estudio e interés es la Revista de Escuelas Normales, hoy Revista Interuniversitaria de Formación del Profesorado. Díez, Pozo y Segura (1988) llevan a cabo un análisis general de la misma, teniendo en cuenta sus características particulares y etapas, así como sus secciones y línea temática. Asimismo, Palomero (2013) presenta la revista haciendo hincapié en sus momentos fundacionales y en los propósitos que esta tenía, mientras que Herrero (1997) la sitúa en el contexto regeneracionista español, presentando una distribución temática de los artículos y a los autores y autoras que publicaron un mayor número de trabajos en el boletín. Es preciso tener en cuenta otras aportaciones, como la de Ortega (2014), quien examina la presencia de Ovide Decroly en los ejemplares de la revista, o el análisis que realiza Herrero (1996) de los contenidos geográficos reflejados en la misma.

Conviene destacar a autores como Martínez (1999) y Merino (2012), que llevan a cabo un análisis descriptivo del Boletín de la Institución Libre de Enseñanza (en adelante, Bile), prestando atención al contexto, origen, objetivos, evolución, directores, secciones y colaboradores; en ambos estudios se incide en el valor del Bile como fuente y recurso de investigación. De igual modo, se han de tener en cuenta otras investigaciones, como la presentada por Villegas e Ibarz (1993) sobre la Revista de Psicología i Pedagogía de Barcelona, la de Montes y Beas (1998), quienes valoran la presencia de Johann Heinrich Pestalozzi en La Escuela Moderna, o el estudio de Aguilera (2005), que analiza los artículos de la Revista de Educación desde una perspectiva de género.

Se observa, por tanto, que existen revistas pedagógicas de referencia que han sido estudiadas a lo largo del tiempo en forma y contenido, mientras que en otras el análisis se dirige a la valoración del tratamiento de temáticas específicas. Escuelas de España no está en ninguna de estas situaciones, pues pese al valor pedagógico que esta tiene, y que así se lo conceden autores como Lozano (1980), Esteban y López (1992), Viñao (1994-1995), Checa (2002), Gómez y Romero (2007) o Aritmendi (2009) nombrándola en sus obras, así como Dueñas y Grimau (2009), quienes hacen continuas alusiones a la revista en su libro sobre la educación del primer tercio del siglo XX en Segovia (España), no existe ninguna aportación que trabaje Escuelas de España, ni de manera general, ni partiendo de alguna temática específica. Este es el motivo por el que a través del este trabajo se pretende realizar un acercamiento a sus características generales, resaltando su valor pedagógico y educativo y su repercusión en el proceso de renovación pedagógica que aconteció en España desde 1929 hasta 1936.

\section{La revista Escuelas de España: origen, propósitos e importancia en el proceso de renovación pedagógica de los centros educativos de España}

Escuelas de España es una revista pedagógica que está compuesta por un total de 44 ejemplares publicados entre enero de 1929 y julio de 1936. Sus principales artífices fueron los maestros segovianos Norberto Hernanz Hernanz, Pablo de Andrés Cobos y David Bayón Carretero, auspiciados por el inspector Antonio Ballesteros Usano.

Segovia fue un núcleo de renovación pedagógica y educativa durante este periodo histórico. No solo constituyó la cuna donde se produjo el nacimiento de Escuelas de España, sino que fue el lugar en el que se originaron los Centros de Colaboración 
Pedagógica (en adelante, CCP), se desarrollaron numerosas cantinas y colonias escolares, se organizaron tres congresos pedagógicos reconocidos a nivel nacional y se fomentó la realización de viajes de estudios por maestros y maestras pensionados por la Diputación de Segovia. (DUEÑAS; GRIMAU, 2009). A todo ello debe añadirse el desarrollo de la Universidad Popular Segoviana, germen de cultura, de aprendizaje y de humanidad, que estuvo abierta a personas de todos los niveles socioculturales y económicos; su labor fue la de "ofrecer al 'pueblo', a través de clases gratuitas, una amplia gama de saberes generales [...], así como una riquísima panorámica de lo más avanzado de la cultura de la época". (VALLES, 2010, p. 39). Con razón advierten Andrés y Mora (2011) que la revista comienza su andadura "en Segovia porque es uno de los mayores focos de inquietud pedagógica y cultural de España [...] Segovia es central en la mejora de la escuela española". (p. 39).

Escuelas de España nació, precisamente, de las ansias de perfeccionamiento que tenían sus entusiastas fundadores, así como de su interés por renovar las escuelas y la educación del país. Norberto Hernanz redacta en sus Memorias (s. f.) cómo se gestó la idea que dio origen a la revista:

\begin{abstract}
Nuestros viajes al extranjero y a diversas provincias españolas, así como las incitaciones recibidas en los congresos, hicieron surgir la idea de crear una revista pedagógica que recogiera y publicara nuestras observaciones y experiencias. [...] La financiamos con nuestros pobres recursos Cobos, Bayón y yo, los tres mosqueteros, como nos llamaban. Una empresa quijotesca que pudo sostenerse durante ocho años... (HERNANZ, s. f., p. 88).
\end{abstract}

Escuelas de España estaba vinculada a la Federación de Trabajadores de la Enseñanza (Fete-UGT), siendo cercana al pensamiento del sector más liberal y renovador del magisterio. (CHECA, 2002). Lozano (1980) expone que la revista "repudiaba todo espíritu de capilla" (p. 196) y que estuvo caracterizada por una notable independencia, situándose en una posición que puede recibir el nombre de "republicanismo pedagógico". Por su parte, Checa (2002) afirma que la publicación pretende "erigirse en revista supraprovincial del Magisterio" (p. 216), sin llegar a tener éxito esta pretensión. No compartimos tal percepción, pues en la revista estuvieron representadas gran parte de las regiones españolas; los ejemplares del primer periodo de publicación nos permiten conocer que llegaron a existir representantes de al menos, 39 provincias $^{1}$, además de un representante de Portugal. Asimismo, existe una gran diversidad en cuanto al lugar de procedencia de quienes escriben en la revista, sin olvidar que a lo largo de su historia se incluyen aportaciones en las que se presentan prácticas educativas, escuelas, así como a maestros y a maestras de diferentes rincones de España. Esta preocupación por tener en cuenta toda la variedad educativa del país se refleja en sus señas de identidad.

Los propósitos de la publicación se recogen en las páginas que dan inicio a cada uno de los números de la revista. En el primer ejemplar (enero, 1929) se destaca como objetivo "dar a conocer las escuelas y los maestros españoles" (p. 3); esta finalidad, unida al tratamiento de la diversidad regional del país mediante el conocimiento mutuo y el

\footnotetext{
1 Álava, Alicante, Ávila, Badajoz, Baleares, Barcelona, Burgos, Cáceres, Cádiz, Castellón, Ciudad Real, Córdoba, Cuenca, Granada, Guadalajara, Guipúzcoa, Huelva, Huesca, Jaén, León, Lérida, Logroño, Lugo, Madrid, Málaga, Murcia, Oviedo, Palencia, Pontevedra, Salamanca, Santander, Sevilla, Teruel, Toledo, Valencia, Valladolid, Vizcaya, Zamora y Zaragoza.
} 
diálogo, se mantiene durante toda la vida de Escuelas de España. Otro de sus propósitos fundamentales consiste en "llevar a los maestros un reactivo para el obrar" (abril, 1930, párr. 2); conceden tanta importancia a este aspecto que desde 1935 incluyen una nueva sección - "El hacer escolar" - con el objetivo de convertir a la revista en "un instrumento eficaz en las manos de todo lector maestro" (diciembre, 1934).

En el número que marca el inicio de la segunda etapa de la publicación (enero, 1934), se incide en su utilización como medio de libre expresión de ideas, conocimientos u opiniones, luchando así contra cualquier intento de censura.

Es que queremos libertad para el pensamiento y para la palabra, y hemos de ofrecer a todos los maestros de España, a todos los profesionales de la enseñanza, vía libre a sus inquietudes y a sus ilusiones. Lo primero que ha de ser ESCUELAS DE ESPAÑA es tribuna libre. Sus páginas quedan de verdad abiertas a todo el que tenga palabra y pensamiento. (párr. 1)

Además de las finalidades recogidas, la más relevante consiste en la intención de transformar y renovar los centros educativos. Escuelas de España "dirigió su esfuerzo a recoger las realidades nuevas y renovadoras de la escuela primaria española" (enero, 1931, párr. 1) y a "facilitar la tarea escolar mejorándola" (enero, 1936, párr. 3). En este sentido, es reseñable el valor que adquiere la revista en el proceso de reconstrucción de la historia de la renovación pedagógica en el primer tercio del siglo. A través del estudio de los autores y autoras que participan en ella, del análisis de sus numerosos temas y mediante el conocimiento de las experiencias renovadoras que se recogen en sus páginas, se puede comprender cómo eran las escuelas y la educación en determinados momentos y lugares de España, y cómo los maestros y las maestras propiciaron prácticas orientadas a la transformación de la escuela, y su adecuación a la sociedad. Heraldo Segoviano ${ }^{2}$ hacía hincapié en que existían en España una gran cantidad de escuelas y de docentes interesantísimos "... que se precisa conocer por todos aquellos maestros que tengan apetencia pedagógica" (p. 5), y que esta "honrosa publicación pedagógica" contribuía a ello. El Magisterio Españoß afirma que Escuelas de España "es un texto selecto, interesante, y el propósito de dar a conocer las Escuelas españolas y digno de aplauso". (p. 326). El periódico La Libertad ${ }^{4}$ recoge que "es un texto selecto de gran enjundia pedagógica, y cuyo propósito principalmente es dar a conocer las escuelas españolas e instituciones complementarias de educación". (p. 2). El Magisterio de Murcia también alude a este propósito:

dedica sus columnas a informar a los maestros españoles sobre las mejores escuelas de España, a reflejar las inquietudes, vacilaciones, ensayos y conquistas, acusados en la honda y rápida transformación por la que está pasando [...] la escuela primaria. (p. 6). ${ }^{5}$

Se trata, pues, de una revista de referencia que contribuye a paliar el vacío que existía hasta el momento en el tratamiento de las escuelas por parte de la prensa

2 Heraldo Segoviano, 6 de enero de 1929. Recuperado de <https://goo.gl/BwP1Aa>.

${ }^{3}$ El Magisterio Español, 30 de enero de 1929. Recuperado de <https://goo.gl/ejHNmG>.

${ }^{4}$ La Libertad, 6 de febrero de 1929. Recuperado de <https://goo.gl/P1CP9w>.

${ }^{5}$ El Magisterio de Murcia, 17 de enero de 1930. Recuperado de <https://goo.gl/vN1yEZ>. 
pedagógica española6 .

La acogida que tuvo Escuelas de España por parte de los y las profesionales de la educación de la época acrecienta el valor que esta adquirió en el proceso de renovación pedagógica de las escuelas. Los ejemplares llegaban a las manos de docentes de todos los niveles educativos y directores escolares que estaban en contacto con las aulas y, por tanto, podrían aplicar prácticas renovadoras en las mismas, así como a las de inspectores de educación que contribuían a impulsar las diferentes iniciativas. Las referencias encontradas en diferentes títulos de prensa histórica muestran esa aceptación. Localizamos varios ejemplos en Heraldo Segoviano": "Sabemos de la buena acogida que han dispensado a la revista, en diferentes provincias de España, y, auguramos una vida próspera y brillante a 'Escuelas de España'”. (p. 5). Este mismo periódico ${ }^{8}$ nos da cuenta del tratamiento que tuvo la revista en otros títulos de prensa:

creemos poder anticipar un éxito seguro y desacostumbrado en las publicaciones pedagógicas. Sabemos que se hacen de muchas provincias importantes pedidos de ejemplares y parece ser que el primer número se agotará rápidamente. La prensa profesional de provincias ha comenzado a ocuparse de 'Escuelas de España', aun antes de conocer la publicación, con señalado cariño; hemos visto calurosos comentarios del proyecto. (p. 5).

Además de los comentarios localizados en ejemplares de este periódico, encontramos críticas positivas, anuncios y recomendaciones de su lectura en otros, locales y nacionales y de diferente naturaleza, como son El Adelantado de Segovia, Universidad y Tierra, El Castellano, El Magisterio Español, La Libertad o El Defensor Escolar. Este éxito, que la revista experimentó desde un primer momento, pudo venir motivado por el equilibrio entre el tratamiento de aspectos pedagógicos teóricos y cuestiones educativas prácticas.

Conviene añadir que entre los redactores de la revista hubo profesores de escuelas normales, escritores y pedagogos de notable influencia y maestros y maestras con una gran sensibilidad hacia la educación y la escuela, muchos de los cuales habían llevado a cabo viajes pedagógicos por España y Europa, pudiendo conocer de primera mano las escuelas más renovadoras del momento, caracterizadas por nutrirse de las ideas educativas de los antecedentes más relevantes de nueva educación, como la Escuela Moderna, la Institución Libre de Enseñanza (en adelante, ILE) o el movimiento de la Escuela Nueva, rompiendo así con el modelo de educación tradicional. Estos viajes de estudio fueron impulsados por la Junta para la Ampliación de Estudios e Investigaciones Científicas (en adelante, JAE) y por diputaciones provinciales. Entre las instituciones educativas visitadas localizamos algunas de reconocida tradición de renovación educativa como el Grupo Escolar Cervantes, el Instituto-Escuela, Baixeras, la Escuela del Mar, la Escuela del Bosque o el Grupo Escolar Joaquín Costa. (MARíN, 1990).

Escuelas de España no fue únicamente una revista. En agosto de 1934 anuncian que comenzarán a realizar actividades editoriales con el propósito de atender a las necesidades y preocupaciones del profesorado, y afirman que la editorial está abierta a "todos los maestros que sepan hacer buenos libros" (párr. 2). Los propios editores son los redactores de las cuatro obras publicadas - quizás porque el proyecto se truncase por el

${ }^{6}$ Heraldo Segoviano, 6 de enero de 1929. Recuperado de <https://goo.gl/BwP1Aa>.

7 Heraldo Segoviano, 6 de enero de 1929. Recuperado de <https://goo.gl/BwP1Aas.

${ }^{8}$ Heraldo Segoviano, 2 de febrero de 1930. Recuperado de <https://goo.gl/nybxge>. 
inicio de la guerra: el último libro se pública en 1936 -, con una sola excepción: la de Ángel Ledesma, pues la primera de las publicaciones, El método de proyectos-Realizaciones, reúne en realidad dos obras, atribuidas a David Bayón y a Ángel Ledesma, respectivamente. Las otras tres son Los clásicos en la escuela de Norberto Hernanz y dos libros de Pablo de Andrés Cobos, Lecciones de arquitectura y Estampas de aldea, este último incluido en el listado de libros prohibidos en la guerra civil española (ANDRÉS; ZUFRIATEGUI; ESTEBARANZ, 2015), y reeditado recientemente (2015) con motivo del octogésimo aniversario de su publicación.

\section{Características generales de Escuelas de España: periodicidad, número de ejemplares, lugares de edición y de publicación}

Escuelas de España se publicó durante ocho años; si bien, su periodicidad y ritmo de publicaciones no fue constante. Identificamos dos etapas (Tabla 01); esta estructuración la establece la propia revista en las páginas iniciales del ejemplar que da inicio a la segunda época (enero, 1934): "estamos de nuevo en camino, otra vez en comunicación con los que anhelan y con los que tienen fe. A caminar. Audacia fue aquel nuestro primer intento y audacísima es esta segunda salida". (p. 1). Autores como Dueñas y Grimau (2009), Gómez y Romero (2007) o la descripción que hace la hemeroteca digital de la Biblioteca Nacional de España (BNE) de la revista, también contemplan esta organización.

Tabla 01 - Etapas de Escuelas de España por periodicidad de publicación.

\begin{tabular}{|c|c|c|c|c|}
\hline PERIODO & LUGAR & PERIODICIDAD & $\begin{array}{c}\text { N.o DE } \\
\text { EJEMPLARES }\end{array}$ & $\begin{array}{c}\text { N.o DE } \\
\text { PÁGINAS }\end{array}$ \\
\hline Enero 1929 - Enero 1932 & $\begin{array}{c}\text { Segovia (hasta } \\
\text { abril de 1931) } \\
\text { Barcelona (hasta } \\
\text { enero de 1932) }\end{array}$ & Trimestral & 13 ejemplares & $\begin{array}{c}\text { Entre } 110 \text { y } 130 \\
\text { por ejemplar }\end{array}$ \\
\hline \multicolumn{7}{|c|}{ SEGUNDA ETAPA DE PUBLICACIÓN } \\
\hline Enero 1934 - Julio 1936 & Madrid & Mensual & 31 ejemplares & $\begin{array}{c}\text { Entre } 50 \text { y } 60 \\
\text { por ejemplar }\end{array}$ \\
\hline
\end{tabular}

Fuente: elaboración propia.

No obstante, si se toman como referencia los lugares de edición y publicación, se definen tres etapas (Tabla 02), coincidiendo con la estructuración que realizan Mora y Hermida (2011).

Tabla 02 - Etapas de Escuelas de España por lugar de publicación.

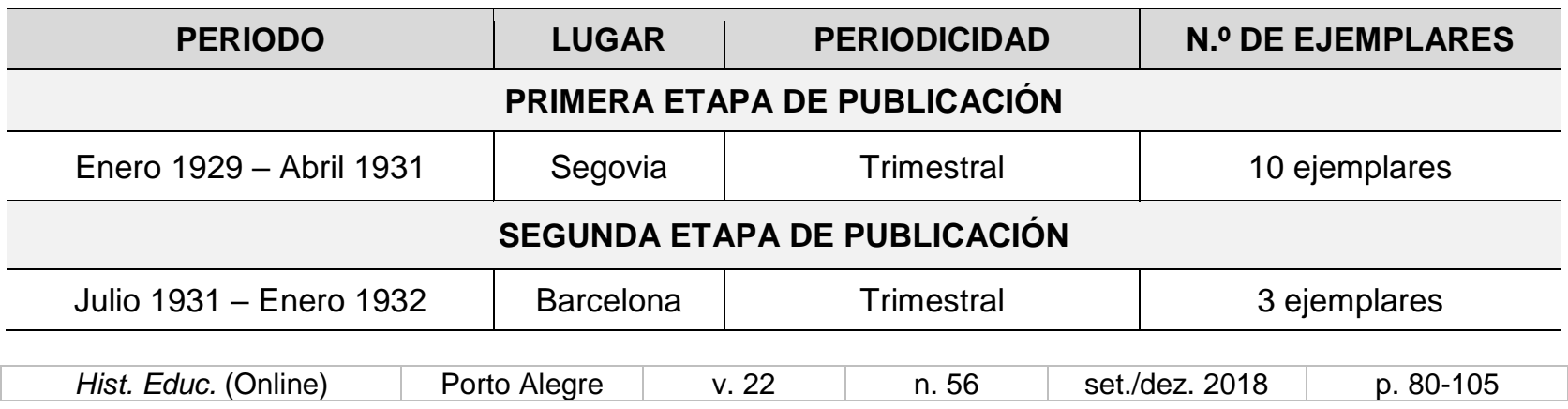




\begin{tabular}{c|c|c|c}
\hline PERIODO & LUGAR & PERIODICIDAD & N.o DE EJEMPLARES \\
\hline \multicolumn{3}{|c}{ TERCERA ETAPA DE PUBLICACIÓN } \\
\hline Enero 1934 - Julio 1936 & Madrid & Mensual & 31 ejemplares \\
\hline
\end{tabular}

Fuente: elaboración propia.

Se trasladó la revista de un lugar a otro en dos ocasiones. El cambio de Segovia a Barcelona pudo ser una consecuencia del traslado de los fundadores de Escuelas de España en 1931 a la ciudad catalana, ya que consiguieron una plaza fija de maestro en el Patronato Municipal (MORA; HERMIDA, 2011); este aviso se refleja en el ejemplar de abril de 1931: "Escuelas de España va, con sus redactores, a Barcelona". (p. 96). La edición de la revista también varió en cada etapa. En el periodo de publicación segoviano fue responsabilidad de la imprenta de Carlos Martín Crespo (Heraldo Segoviano), mientras que los ejemplares de Barcelona se editaron en la imprenta Athenea de Alegret y Lluch. Por su parte, en la época de publicación madrileña, colaborarían con Imprenta - Encuadernación Yagües hasta mayo de 1934, con la Editorial Plutarco hasta marzo de 1935 y con Tipografía López hasta junio de 1935, momento en el que se encargaría de ello Talleres Tipográficos de Cleto Vallinas.

Cabe destacar la inactividad de la revista entre enero de 1932 y enero de 1934 debido a la insuficiente financiación de la que disponían: el ejemplar de enero de 1932 anuncia que "Escuelas de España se publicará de ahora en adelante sin compromiso de regularidad. Cuando tengamos dinero para hacer un número, saldrá el número y se pondrá a la venta". (p. 3-4).

\section{Características materiales y aspectos económicos}

La maquetación de Escuelas de España se asemeja a la de otras publicaciones pedagógicas de la época. Los ejemplares de la primera etapa tienen unas dimensiones de $19.5 \mathrm{~cm}$ de alto por $13 \mathrm{~cm}$ de ancho, mientras que en los de la segunda etapa aumenta, midiendo $21.5 \mathrm{~cm}$ de alto por $16 \mathrm{~cm}$ de ancho. Las páginas de los primeros ejemplares se unen mediante un cordel, mientras que posteriormente se integran dos grapas. El formato, por tanto, no varió sustancialmente en el transcurso de los años, aunque la presentación de la revista constituía un aspecto de importancia para sus directores; en una de las cartas escritas por Norberto Hernanz a David Bayón el 22 de enero de 1934 (recogidas por Mora y Hermida - 2011 -), aparece el deseo de mejora de la maquetación:

Hay que mejorar el papel de la revista y la encuadernación. De ningún modo dar sensación de pobreza. Hay que mejorar las cubiertas. Sea corta o larga su vida que salga con dignidad hasta en la forma más externa y superficial. (p. 104).

Se observa un cambio sustancial en el empleo de diferentes lenguajes y en el tamaño y tipo de letra; hasta 1934, que es cuando comienza la segunda etapa de Escuelas de España, la revista únicamente contenía textos escritos, mientras que a partir de esta fecha se incorporarán imágenes, dibujos, fotografías o pentagramas (Figura 01). 


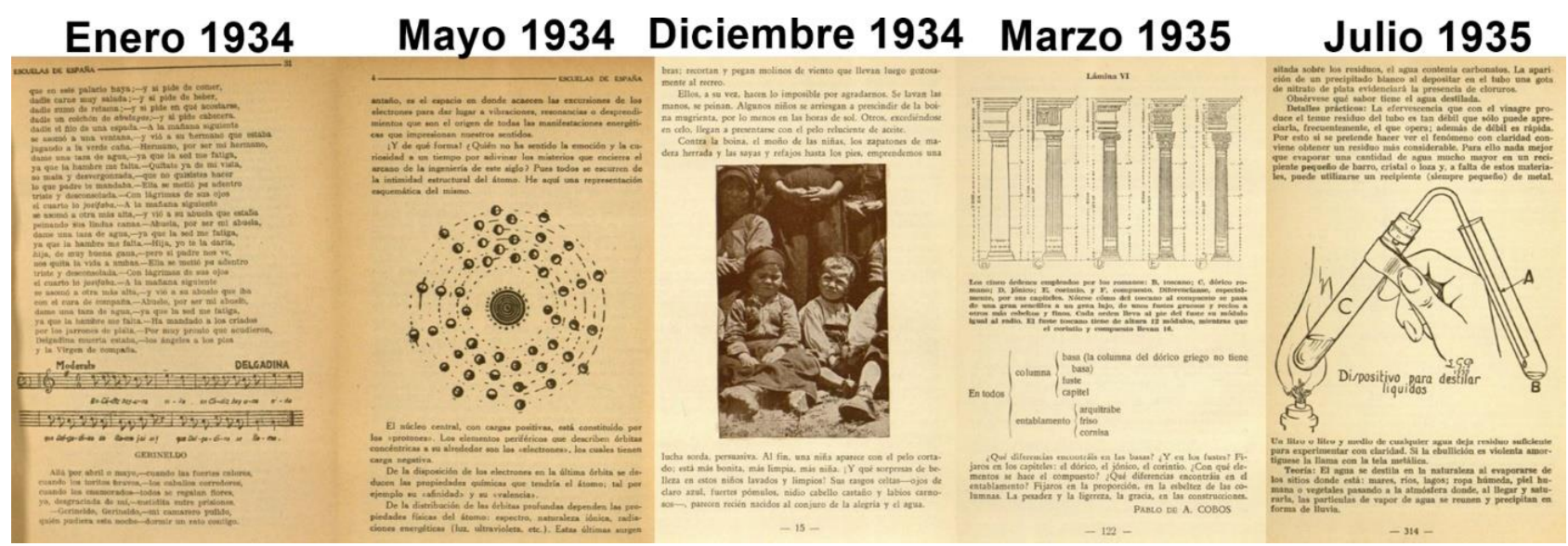

Figura 01 - Fotografías y dibujos en Escuelas de España.

Fuente: Escuelas de España (enero, 1934; mayo, 1934; diciembre, 1934; marzo, 1935; julio, 1935).

Desde entonces, el cambio más destacable es la modificación en la numeración de las páginas; hasta 1935 cada ejemplar tiene su propia paginación, mientras que en los números de 1935 y 1936 la numeración se inicia cada año, de modo que en el primer caso llega a abarcar 588 páginas (diciembre, 1935) y en el segundo caso 336, teniendo en cuenta que la revista llegó a su fin en julio de 1936.

Durante la primera etapa cada ejemplar tenía un precio de dos pesetas, mientras que en la segunda época este era de 1.25 pesetas; partiendo de que los ejemplares del primer periodo eran más amplios en su extensión que los del segundo, las tarifas se mantuvieron ciertamente homogéneas. De igual modo, existían suscripciones anuales con ofertas para los lectores, apareciendo la opción de "suscripción semestral" desde la segunda etapa de publicación. Las opciones de pago para los anunciantes también son distintas en las dos etapas de Escuelas de España (Figura 02 y 03).

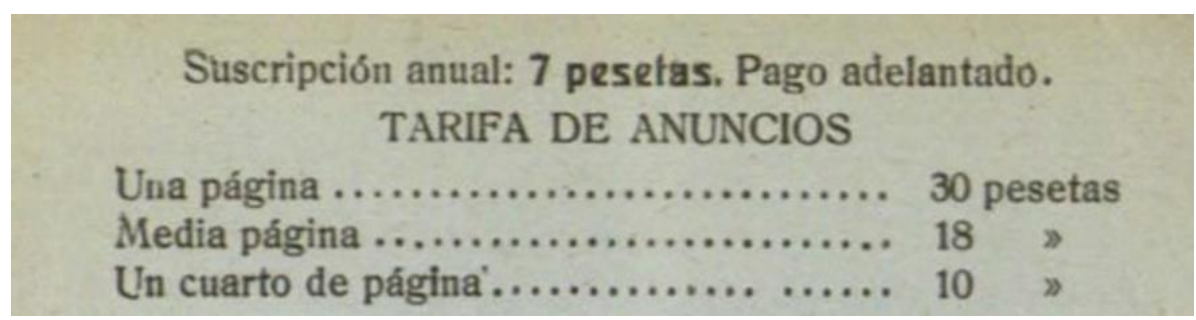

Figura 02 - Tarifas de Escuelas de España en la primera etapa de publicación.

Fuente: Escuelas de España (octubre, 1929). 
PRECIOS DE SUSCRIPCION

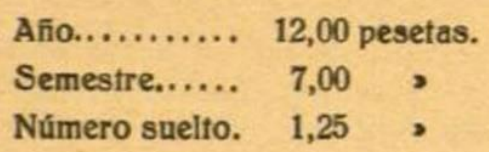

Las suscripciones se abonan por adelantado. Dirfjase toda la correspondencia a sus oficinas: Aguila, 3 pral.-Madrid.

\section{TARIFA DE ANUNCIOS}

\begin{tabular}{|c|c|c|c|}
\hline & $\begin{array}{l}12 \text { inserciones } \\
\text { Por Inserción }\end{array}$ & $\begin{array}{l}6 \text { inserciones } \\
\text { Por inserción }\end{array}$ & 1 inserción \\
\hline Una plana ......... & 75 ptas. & 85 ptas. & 100 ptas. \\
\hline Media............ & $40=$ & 50 , & 75 , \\
\hline Cuarto....... & $25=$ & $35>$ & 50. \\
\hline
\end{tabular}

Figura 03 - Tarifas de Escuelas de España en la segunda etapa de publicación.

Fuente: Escuelas de España (abril, 1934).

\section{Estructura y secciones de la revista}

La estructura de Escuelas de España guarda cierto paralelismo en todos sus ejemplares y, según Lozano (1980), es similar a la de la mayoría de las revistas pedagógicas de la época, existiendo algunos cambios poco sustanciales en determinados números. Este autor señala que la revista tiene la siguiente estructura:

Estudios doctrinales sobre los problemas de educación. Amplia selección dedicada al examen del hacer escolar.

Estudios sobre asuntos fundamentales en otros dominios de la cultura. Información, glosa y crítica de la actualidad pedagógica.

Reseña y crítica de libros.

Noticias de España y del extranjero. (LOZANO, p. 196).

A continuación, se realiza un análisis de la estructura de forma más detallada partiendo de Ortiz (2017) y de nueva información extraída de los ejemplares.

a) Portada con el título de la revista, el número - a partir de 1930, también el número de años que lleva publicándose -; lugar, mes y año de publicación; índice o sumario con el nombre de los artículos y sus autores. A partir de 1934 - segunda etapa - se comienza a contabilizar de nuevo el número de la revista (Figura 04). 


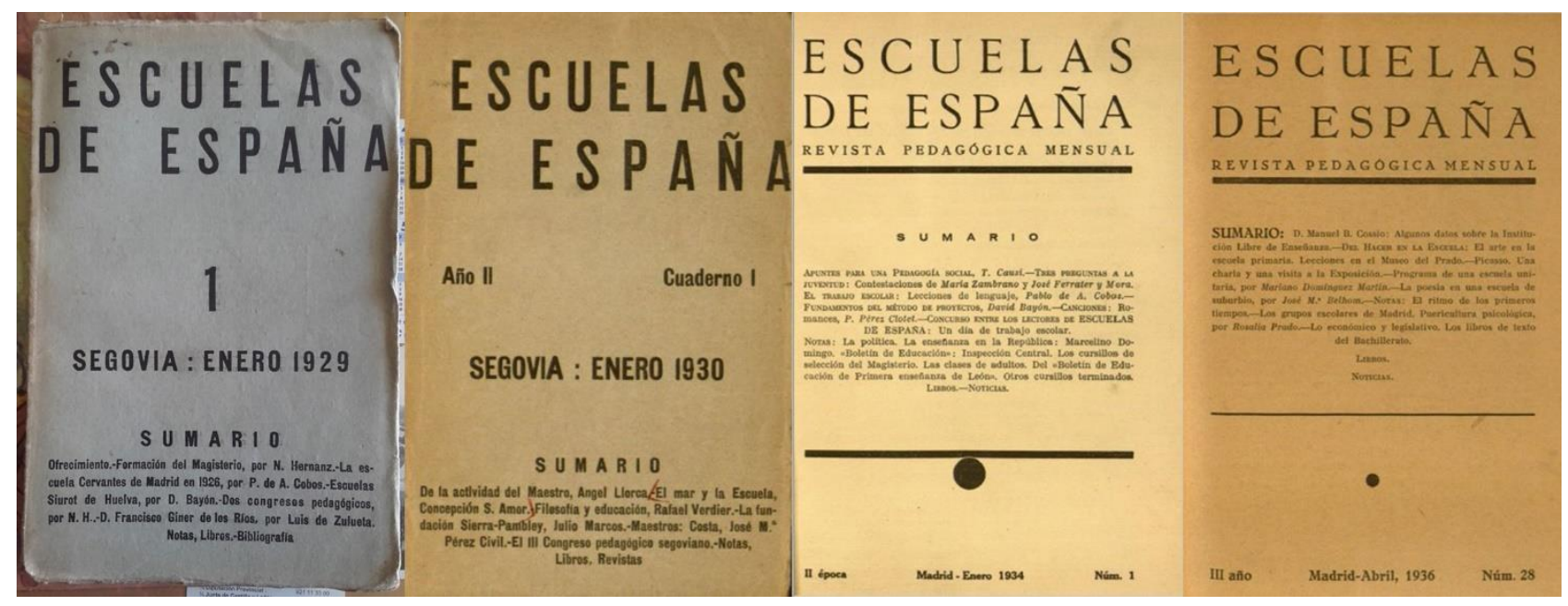

Figura 04 - Portadas de Escuelas de España.

Fuente: Escuelas de España (enero, 1929; enero, 1930; enero, 1934; abril, 1936).

b) Presentación de las señas de identidad de Escuelas de España y de cada número en particular. El formato de este texto será diferente en la etapa de publicación segoviana, en los ejemplares de 1934 y en los de 1936 (Figura 05). Además, en algunos números los autores redactan un texto explicando la finalidad del ejemplar (enero, 1931; abril, 1931; julio, 1931; enero, 1932; agosto 1934) y en otros no se reflejan los propósitos de Escuelas de España, como es el caso de las publicaciones de 1935 o los números publicados en octubre de 1931 y en febrero y marzo de 1934.

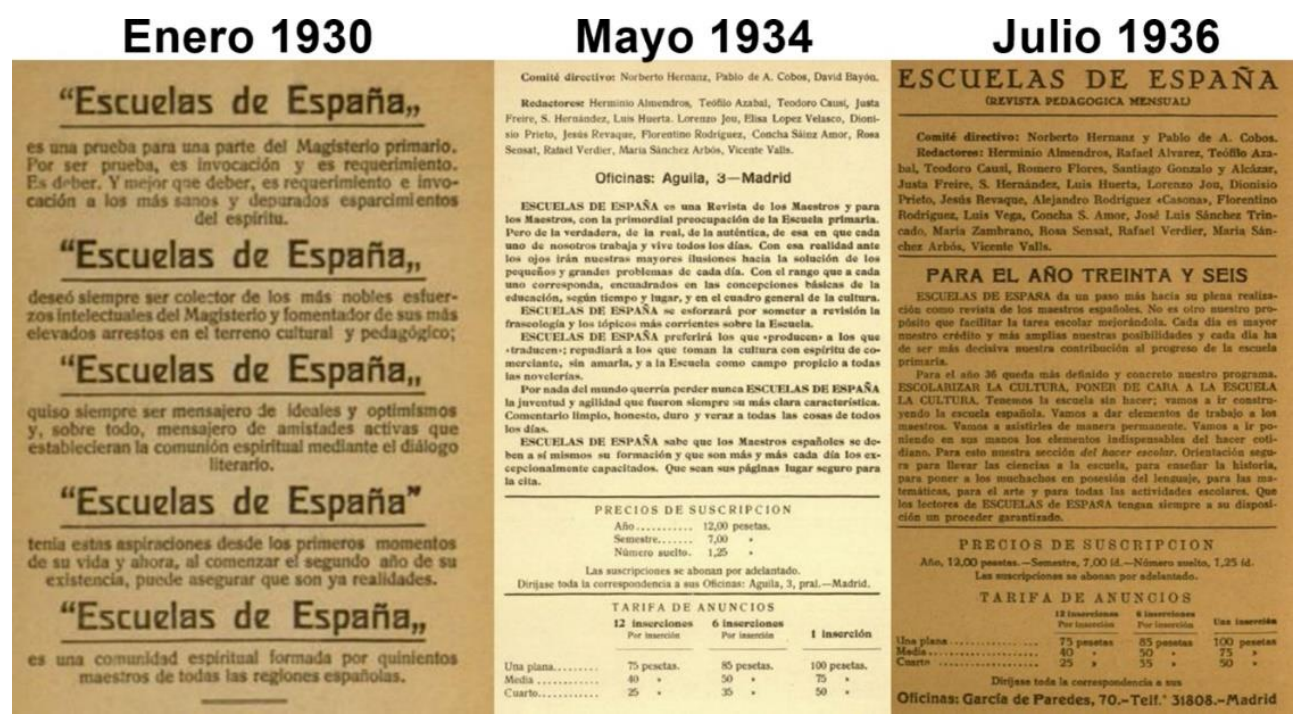

Figura 05 - Ejemplos de señas de identidad de Escuelas de España.

Fuente: Escuelas de España (enero, 1930; mayo, 1934; julio, 1936).

c) Una segunda portada con los mismos datos que la primera, menos el índice, seguida de una hoja en blanco con los datos de la imprenta (hasta 1934).

d) Secuencia de artículos sobre temas de educación y de la vida pedagógica de la época. Constituye el núcleo fundamental de la revista. Se publicaron 247 artículos a lo largo de la vida de Escuelas de España (16 sin firmar); cada uno de los ejemplares tienen una media de seis aportaciones. Aunque no se observa una división continua y estática de 
los artículos en "secciones", se pueden identificar algunos paralelismos entre varios ejemplares. En ocho de ellos existe un espacio destinado a reconocer el excelente trabajo de figuras señeras de la educación, a quienes denominan "maestros", entre los que encontramos los dedicados a Santiago García Rivero (julio, 1929, abril, 1930 y enero, 1931), Miguel de Unamuno y Martín Chico Suárez (octubre, 1929), Joaquín Costa Martínez (enero, 1930), Alice Pestana (octubre, 1931), Ángel Llorca i García (enero, 1932) o Manuel Bartolomé Cossío (abril, 1929). En recuerdo a este último se escriben, además, siete artículos desde el ejemplar de octubre de 1935 -con motivo de su fallecimiento- hasta el final de Escuelas de España. Asimismo, se localiza un número extraordinario dedicado a Félix Lope de Vega (mayo, 1935) y diferentes artículos que rememoran la labor de personas como José Ortega y Gasset o Francisco Giner de los Ríos.

"El hacer escolar" o "Del hacer en la escuela" es otra sección que se introduce desde julio de 1935 hasta julio de 1936 -14 artículos-, con el objetivo de hacer de la revista un recurso e instrumento de enseñanza y aprendizaje que los docentes de primera enseñanza pudiesen emplear para planificar su práctica educativa. Igualmente, se encuentra la sección "Informaciones" en tres ejemplares, destinada al tratamiento de cuestiones pedagógicas concretas.

Por otra parte, en su interés por lograr la colaboración con otras regiones de España, recogen artículos redactados por profesionales de la educación de diferentes lugares a lo largo de toda la historia de la revista e incluyen monográficos específicos de algunas regiones españolas: Aragón (abril, 1930), Asturias (julio, 1930), Cataluña (octubre, 1930) y Segovia (abril, 1931), además del número de enero de 1931 que contiene cuatro artículos elaborados por personas del País Vasco.

Es preciso destacar los dos concursos que convoca la revista. En enero de 1934 se anuncia el primero, por el que los lectores y las lectoras pueden participar escribiendo un artículo acerca de un día de trabajo escolar; estas aportaciones se publicaron en los números sucesivos de la revista, hasta octubre de 1934 que es cuando se convoca el segundo concurso, dedicado al tratamiento de la escuela rural, urbana y unitaria.

e) "Notas": pequeños espacios destinados a comentar acontecimientos o cuestiones de interés de la realidad educativa y pedagógica. Hay 204 notas, 177 sin firmar, con una media de cinco notas por ejemplar.

f) "Libros y revistas": se realiza una crítica de varias publicaciones nacionales e internacionales, analizando brevemente su contenido y destacando su riqueza pedagógica. Hay un total de 154 críticas de libros (91 sin firmar), existiendo una media de cuatro reseñas por ejemplar, pues en los números de agosto de 1934, diciembre de 1935 y febrero y marzo de 1936 se suprime esta sección por "necesidades de ajuste".

g) "Noticias" nacionales y del extranjero desde la segunda etapa de publicación.

En todos los ejemplares se incluye un folio con la siguiente información: a) La tarifa de la revista y de los anuncios. b) La dirección de la correspondencia: a Pablo de Andrés Cobos (San Ildefonso) en 1929; a Norberto Hernanz (San Millán: Segovia y Calle de las Cortes: Barcelona) hasta octubre de 1931 y a David Bayón (Diputación: Barcelona) en enero de 1932. Desde enero de 1934 la correspondencia se dirigía a las Oficinas Águila, 3 
(Madrid) ${ }^{9}$, de las cuales se trasladaron en enero de 1936 a Oficinas García de Paredes, 70 (Madrid). Durante la primera etapa de publicación esta información se encuentra al final de Escuelas de España (Figura 02) y se incluyen, también, los representantes de la revista: el número de representantes se incrementa desde 22 en 1929 hasta llegar a 34 en enero de 1932. Desde enero de 1934 los datos de la tarifa y de la correspondencia se incluyen al inicio de cada número (Figura 03).

\section{Comité de redacción}

El Comité Directivo estaba formado por los tres fundadores de la revista: Norberto Hernanz, Pablo de Andrés Cobos y David Bayón.

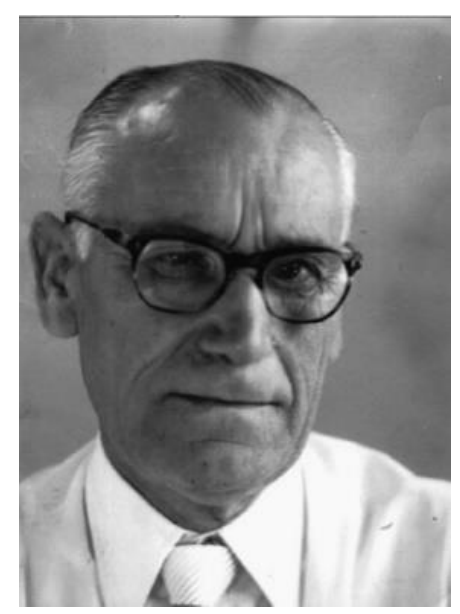

Figura 06 - Norberto Hernanz Hernanz.

Fuente: Mora y Hermida (2011).

Norberto Hernanz ${ }^{10}$ (Figura 06) nació en 1891, en Sanchopedro (Segovia). Estudió en la Escuela Normal de maestros y en la Escuela Superior de Magisterio de Madrid (1914). Fue el inspirador de importantes iniciativas educativas y pedagógicas, siendo una de las más relevantes la creación de los CCP junto al maestro Lorenzo del Amo o los congresos pedagógicos provinciales, que impulsó con Pablo de Andrés Cobos .

Fue maestro en Torre Val de San Pedro (Segovia) desde 1916. En 1931 consiguió una plaza en el Patronato escolar de Barcelona; allí ejerció en la escuela Baixeras. (GARCÍA, 2008). Su actividad como maestro se caracterizó por un fuerte carácter renovador: potenciaba el aprendizaje colaborativo, defendía la enseñanza individualizada como él mismo denomina- y favorecía el contacto con las familias, señalando que la escuela "no debe ser una institución aislada del medio social y físico en que vive". (HERNANZ, s. f., p. 84).

Con la llegada de la guerra civil, fue depurado de sus funciones y suspendido de empleo y sueldo. En 1944 sería nuevamente rehabilitado como maestro y destinado a Mayals (Lérida), aunque no abandonó Barcelona. Primeramente, se incorporó en un centro educativo de frailes y más tarde en otro colegio; por último, se dedicó a la Academia Práctica

\footnotetext{
${ }^{9}$ Residencia de David Bayón.

10 Información obtenida de Mora y Hermida (2011), de Hernanz (s. f.) y del testimonio de su nieto, Norberto García Hernanz.
} 
hasta su jubilación. Norberto falleció en 1981.

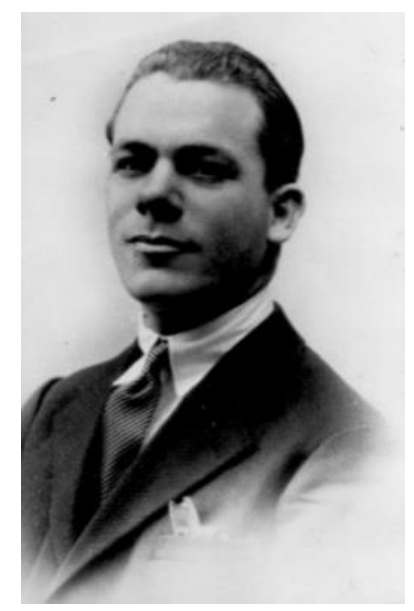

Figura 07 - Pablo de Andrés Cobos.

Fuente: Estebaranz (1, marzo, 2016).

Cobos (Figura 07) nació el 1 de junio de 1899 en La Cuesta (Segovia). Su conexión con el ámbito rural, posiblemente, le hizo formar parte activa de las Misiones Pedagógicas entre 1931 y 1933. Estudió en la Escuela Normal de Segovia y adquirió el título de maestro de primera enseñanza en 1920, aprobando la oposición libre ese mismo año e incorporándose en 1921 a la escuela de Valdepeñas (Ciudad Real). En 1925 se trasladó a San Ildefonso (Segovia); en la escuela graduada de niños impulsó la Sociedad Infantil (1926), basándose en el grupo escolar Baixeras de Barcelona. (DUEÑAS; GRIMAU, 2009).

En 1931 logró una plaza como maestro en Barcelona, pero pronto regresó a Madrid; según Mora y Hermida (2011), "quizá fuera por su fuerte vinculación a Segovia que nunca interrumpió". (p. 98). En 1933 fue director del Orfanato Nacional del Pardo y en 1934 obtuvo una plaza como director del grupo escolar Claudio Moyano (Madrid). Se convertiría en su última vivencia como maestro dentro de la enseñanza reglada, pues en 1936 fue encarcelado, acusado por "conspiración para la rebelión". (ANDRÉS; MORA, 2011).

Tras su salida de prisión en 1940 trata de sacar adelante a su familia con la creación de un taller de prendas y dando clases de oposiciones; llegó a formar su propia academia. Cobos no se separó de su pasión, la enseñanza, ni tampoco dejó de escribir en revistas y libros, llegando a fundar su propia editorial: "ancos"11, y participando en la editorial y la revista Ínsula hasta su fallecimiento (1973).

${ }^{11}$ Se corresponde con el acrónimo de sus apellidos (Andrés Cobos). 


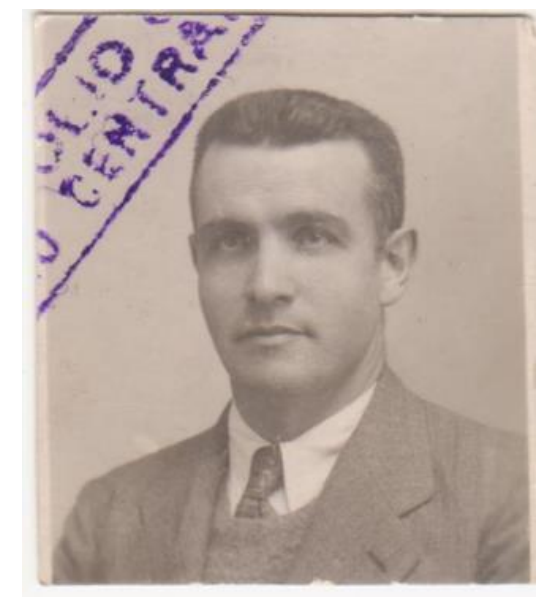

Figura 08 - David Bayón Carretero .

Fuente: Archivo personal de la familia de David Bayón.

David Bayón Carretero (Figura 08) fue maestro de primera enseñanza en Riaza y en Cuéllar, municipios de Segovia. (MORA; HERMIDA, 2011). Fue director del grupo escolar Villalpando (Segovia) en 1961, en el que se jubiló. (FERNÁNDEZ, s. f.). En la revista que se editó en el centro a causa de la celebración del quincuagésimo aniversario (2006), se indica que "era un director escolar de gran prestigio y muy avanzado en su época".

Bayón obtuvo una beca individual en 1924 por la JAE que le permitió viajar a Francia, Bélgica y Suiza; de estos viajes nacieron sus numerosos escritos sobre política educativa, organización escolar y métodos de enseñanza. (MARíN, 1990). Al igual que Hernanz y Cobos, también fue pensionado por la Diputación Provincial de Segovia; Cobos en 1926 y Bayón y Hernanz en 1927 para España, y los tres juntos en 1929/1930 para recorrer escuelas de interés de otros países europeos. (DUEÑAS; GRIMAU, 2009). Estas experiencias motivaron a los maestros a exponer conferencias, a redactar publicaciones pedagógicas, y constituyeron un punto clave en la consolidación de su formación pedagógica y filosófica. Asimismo, de las visitas de Cobos a las escuelas españolas nació su obra Un viaje por las escuelas de España (1927).

\section{Otros autores y autoras}

Escuelas de España fue posible gracias a las aportaciones de 134 personas, entre las que identificamos a maestros y maestras de primera enseñanza, inspectores de educación, profesores de escuelas normales, directores de grupos escolares de referencia, pedagogos, escritores y otras figuras vinculadas a la educación.

Existe una gran diferencia entre la participación femenina y la masculina, pues 115 son hombres y tan solo 19 son mujeres. A comienzos del siglo XX la "equiparación numérica de maestros y maestras era ya un hecho" (BALLARín, 2016) en los primeros niveles educativos. Este hecho contrasta con el propósito de la publicación de representar a toda la comunidad educativa. Aunque nos situamos en un contexto en el que la mujer empezaba a tener una mayor representación en la sociedad, en Escuelas de España existe una perspectiva androcéntrica. Son pocas las mujeres participantes si lo abordamos desde un punto de vista puramente cuantitativo; no obstante, adquiere una dimensión más valiosa si dirigimos la mirada a las características humanas de estas mujeres, la mayoría situadas en el colectivo de "maestras republicanas", quienes encarnaban el modelo de mujer 
independiente, entusiasta y transgresora, símbolo del ideal educativo de la Segunda República, basado en la defensa de una educación pública, laica y coeducativa, bajo los principios de igualdad y solidaridad. (SÁNCHEZ, 2012).

Del total de 605 aportaciones - artículos, críticas de libros y notas -, 33 están escritas por mujeres y 288 por hombres, teniendo en cuenta que 284 producciones aparecen sin firmar (Figura 09). El contenido de gran parte de estas aportaciones nos hace deducir que han sido escritas por el Comité de Redacción; no obstante, como no aparece reflejado de forma explícita, serán tratadas como contribuciones "sin firmar", sin perder la vista de que cabe la posibilidad de que gran parte de las mismas pueden haber estado redactadas por hombres.

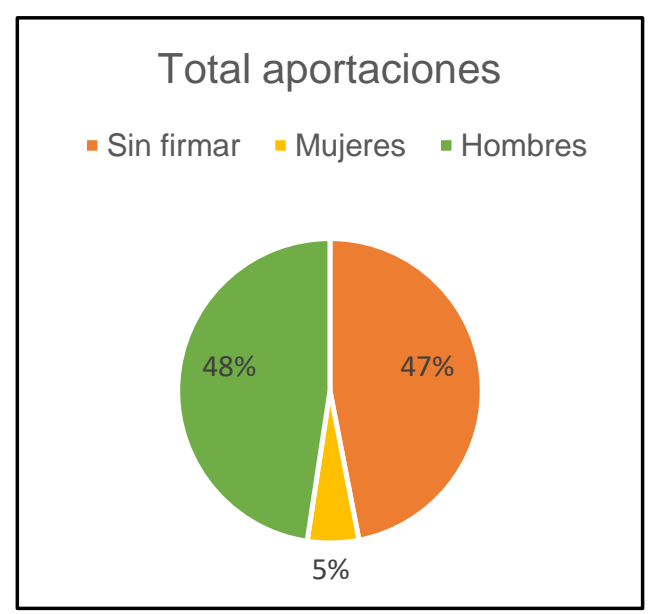

Figura 09 - Producciones totales.

Fuente: elaboración propia desde Escuelas de España.

Esta diferencia se observa en la redacción de artículos. 28 de las 33 aportaciones realizadas por mujeres son artículos, núcleo fundamental de la revista. Por su parte, ninguna mujer colaboró en la elaboración de críticas de libro y tan solo cinco notas están elaboradas por mujeres: tres de María Sánchez Arbós y dos de Rosalía Prado.

Por otro lado, se encuentran diferencias en el grado de participación de cada persona. 82 colaboran con una sola aportación y 40 personas firman entre dos y cinco contribuciones, mientras que existe un pequeño núcleo más productivo - 12 personas que ha escrito seis trabajos o más (Figura 10). 


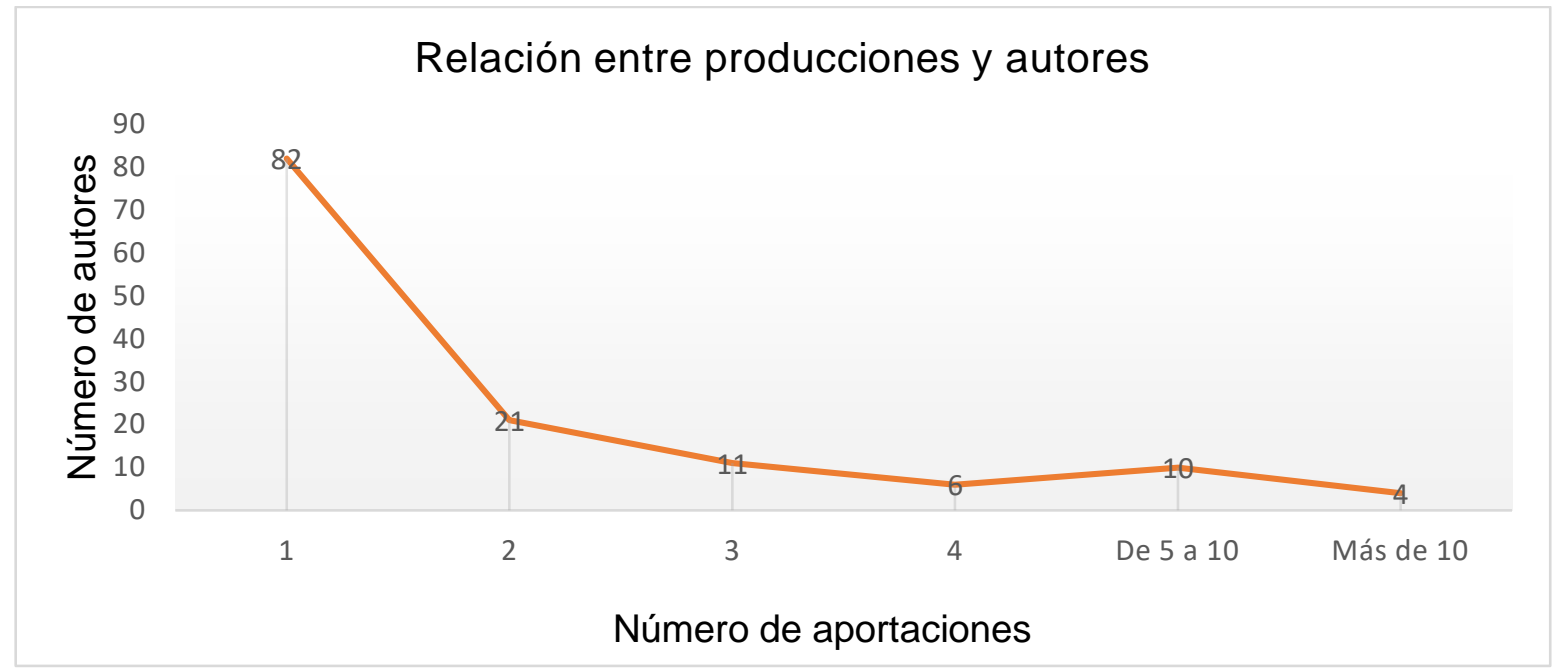

Figura 10 - Relación entre número de aportaciones y autores.

Fuente: elaboración propia desde Escuelas de España.

Los tres fundadores de Escuelas de España son quienes más publican en la revista; Hernanz aporta 26 producciones (19 artículos, cinco reseñas de libro y dos notas), Cobos participa con 28 (15 artículos y 13 reseñas) y Bayón tiene 27 (12 artículos, 12 críticas de libro y tres notas). Además de ellos, existe otro grupo de redactores con un número considerable de aportaciones como Julio Sánchez López con 11 trabajos, Luis Huerta Naves con nueve, Lorenzo Gascón Portero, Rafael Verdier Vázquez, Teófilo Azabal Molina y Mariano Domínguez Martín con ocho y Teodoro Causí Casaus, Alejandro Manzanares Beriain y María Sánchez Arbós con siete contribuciones. Además de esta última, las mujeres que más presencia tienen en la revista son Justa Freire Méndez (cuatro aportaciones) y María Baldó de Torres (tres aportaciones).

La revista, como ya indicaba Lozano (1980), prestó atención a diversos temas educativos, pero especialmente se dedicó al conocimiento de la escuela primaria. Por este motivo el grupo de redactores más amplio está formado por maestros y maestras de escuela. Entre ellos, numerosas personas fueron pensionadas por la JAE (MARíN, 1990) o por otras instituciones para realizar viajes a escuelas de España y del resto de Europa, como Luis Huerta Naves, fundador de la revista Enseñanza Moderna en 1912 (DIEGO, 2006) y autor de cuatro de los libros que fueron prohibidos durante la Guerra Civil Española12; Justa Freire Méndez y Elisa López Velasco, maestras del Grupo Escolar Cervantes (Madrid) y colaboradoras en las Misiones Pedagógicas; o Concepción SáinzAmor Alonso, preocupada por la mejora de la escuela rural y la coeducación, escritora asidua del Bile (ALONSO, 2009) y presidenta del Congreso Pedagógico de la Granja ${ }^{13}$ (Segovia) en 1931. Muchos de estos docentes, además de otros como Fernando Norberto Cerezo o Teodoro Causí Casaus colaboraban con Fete-UGT y representan al sector más renovador de la educación en este momento histórico.

María Baldó de Torres, maestra colaboradora de la revista, dirigió el primer grupo escolar femenino del Patronato Escolar de Barcelona (La Farigola), fue directora del Lyceum Club y vicedirectora del Comité Ejecutivo de la Unión de Mujeres de Cataluña

12 Dicción y redacción, Las artes en la escuela, Salud y Derecho usual español. ABC (Sevilla, 1938): Disponible en: <https://goo.gl/9sDo7L>.

${ }_{13}$ Segovia Republicana, Número 66, 19 de julio de 1931. Disponible en: <https://goo.gl/wgqPq7>. 
(Mellado, febrero, 2017). Por otra parte, destacamos al maestro Francisco García Mochales, secretario del patronato de Misiones Pedagógicas ${ }^{14}$ o a Antonio Benaiges Nogués, impulsor de las técnicas de Freinet en su escuela (Bañuelos de Bureba, Burgos).

Por otra parte, existe un amplio número de docentes que fueron, además, directores de escuela, lo que podría explicar la circulación que la revista tuvo en las escuelas del país y el conocimiento y acceso que los docentes tuvieron a la misma; no obstante, para poder afirmar esto es necesaria una investigación más exhaustiva dirigida a analizar el papel de los directores escolares en la difusión de Escuelas de España. Entre ellos destacamos a Ángel Llorca i García (Grupo Escolar Cervantes), aprendiz del pedagogo Manuel Bartolomé Cossío e influenciado por la ILE, es considerado como uno de los grandes impulsores de la renovación pedagógica en España; en un ejemplar de Escuelas de España (enero, 1929) Cobos afirma: "creo que el señor Llorca es el más completo de los maestros que tenemos en España". (p. 34). Por su parte, el director de escuela Félix Martí Alpera (Grupo Escolar Baixeras) dedicó sus esfuerzos a defender la identidad de la escuela rural.

Los maestros - inspectores de primera enseñanza también están presentes en Escuelas de España. Algunos fueron pensionados por la JAE, como Antonio Ballesteros, Inspector Central del Ministerio de Instrucción Pública durante la Segunda República. (MARÍN, 1990). Colaboró con diversas instituciones escolares y fue el artífice de la Asociación de Amigos de la Escuela en Segovia. (DUEÑAS; GRIMAU, 2009). Otro de ellos es Luis Álvarez Santullano, vocal de la JAE desde su fundación (1907). Colaboró con Cossío en un proyecto de reforma de las escuelas normales y fue vocal-secretario de las Misiones Pedagógicas. (GONZÁLEZ, 2010). Otros inspectores que escriben en la publicación son Gervasio Ramos Álvarez, Santiago Hernández Ruiz y Herminio Almendros, quien tuvo una gran influencia de la ILE y de las corrientes pedagógicas europeas, promovió reformas escolares innovadoras, participó en las Misiones Pedagógicas y colaboró en el movimiento de renovación pedagógica de Cataluña. (BLAT, 1998).

No podemos dejar de mencionar a los profesores de escuelas normales Alejandro de Tudela y Lorenzo Gascón Portero, ni al docente de la Escuela de Estudios Superiores del Magisterio, Luis de Zulueta y Escolano, también influenciado por la ILE.

El contenido puramente didáctico-pedagógico y práctico de la revista se ve completado por reflexiones y aportaciones teóricas pedagógicas (Cossío o Isidoro Enríquez Calleja), filosóficas (José Gaos y González-Pola, María Zambrano Alarcón o José Ferrater y Mora), lingüísticas y literarias (Mariano Quintanilla Romero, Enrique Azcoaga o José Luis Sánchez-Trincado) e histórico-artísticas (Emilio Camps Cazorla, Enrique Lafuente Ferrari, Juan Uría Ríu, Francisco Javier Cabello Dodero o Elías Ortiz de la Torre). Además de estos, colaboran en Escuelas de España profesionales como Carlos Sáinz de los Terreros, pediatra que participa en la selección de los estudiantes para las colonias escolares o el periodista Luis Nicolás Bello Trompeta que visita numerosas escuelas del país, dando origen a su obra Viaje por las escuelas de España (1926).

\section{Contenidos principales}

Escuelas de España fue un fiel reflejo de la realidad educativa en España entre 1929 y 1936. La diversidad de autores junto con la evolución y el propósito de la revista

${ }^{14}$ Las Misiones Pedagógicas. Disponible en: <https://goo.gl/cDJv4i>. 
evidencia la variedad temática que se puede encontrar en la misma. Aparecen contenidos de la pedagogía general, de organización y enseñanza de las diferentes disciplinas, de metodología educativa, así como biografías y problemas y reflexiones de la realidad educativa de la época. Con la finalidad de concretar estos contenidos, se establecen las siguientes categorías, que tienen su origen en la intencionalidad principal de cada artículo así como en el tema predominante de cada uno de ellos (Cuadro 01).

Cuadro 01 - Contenidos de Escuelas de España.

\begin{tabular}{|c|c|c|c|}
\hline \multicolumn{4}{|c|}{ CONTENIDOS DE ESCUELAS DE ESPAÑA } \\
\hline \multirow{5}{*}{$\begin{array}{l}\text { CONCEPCIÓN DE LA } \\
\text { ESCUELA Y DE LA } \\
\text { EDUCACIÓN }\end{array}$} & \multicolumn{3}{|c|}{ El papel de la escuela y de la educación en la sociedad } \\
\hline & \multicolumn{3}{|c|}{ Reformas educativas } \\
\hline & \multicolumn{3}{|c|}{ Defensa de la escuela rural } \\
\hline & \multicolumn{3}{|c|}{ Defensa de la educación pública } \\
\hline & \multicolumn{3}{|c|}{ La escuela y la religión } \\
\hline \multirow{8}{*}{$\begin{array}{l}\text { INICIATIVAS DE } \\
\text { RENOVACIÓN } \\
\text { PEDAGÓGICA }\end{array}$} & \multicolumn{3}{|c|}{ Grupos escolares renovadores } \\
\hline & \multicolumn{3}{|c|}{ Colonias escolares } \\
\hline & \multicolumn{3}{|c|}{ Cantinas y roperos } \\
\hline & \multicolumn{3}{|c|}{ Centros de Colaboración Pedagógica } \\
\hline & \multicolumn{3}{|c|}{ Congresos pedagógicos } \\
\hline & \multicolumn{3}{|c|}{ Universidades Populares } \\
\hline & \multicolumn{3}{|c|}{ Viajes por las escuelas de España y del resto de Europa } \\
\hline & \multicolumn{3}{|c|}{ Las Misiones Pedagógicas } \\
\hline \multirow{12}{*}{$\begin{array}{l}\text { PROTAGONISTAS DE LA } \\
\text { EDUCACIÓN }\end{array}$} & \multirow{3}{*}{ El alumnado } & \multicolumn{2}{|c|}{ Respeto y amor a la infancia } \\
\hline & & \multicolumn{2}{|l|}{ Autonomía y libertad } \\
\hline & & \multicolumn{2}{|c|}{ El niño y sus derechos } \\
\hline & \multirow{8}{*}{ El docente } & \multicolumn{2}{|c|}{$\begin{array}{l}\text { Maestros y maestras de referencia en la } \\
\text { renovación pedagógica }\end{array}$} \\
\hline & & \multicolumn{2}{|c|}{ Concepción de la figura del docente } \\
\hline & & \multicolumn{2}{|c|}{ Sistema de oposiciones } \\
\hline & & \multirow{2}{*}{$\begin{array}{l}\text { Perfeccionamiento } \\
\text { docente }\end{array}$} & Formación inicial \\
\hline & & & Formación permanente \\
\hline & & \multicolumn{2}{|l|}{ Directores escolares } \\
\hline & & \multicolumn{2}{|c|}{ Inspectores de primera enseñanza } \\
\hline & & \multicolumn{2}{|l|}{ Las maestras } \\
\hline & Las familias & & \\
\hline
\end{tabular}




\begin{tabular}{|c|c|c|}
\hline \multicolumn{3}{|c|}{ CONTENIDOS DE ESCUELAS DE ESPAÑA } \\
\hline \multirow{18}{*}{$\begin{array}{l}\text { LA RENOVACIÓN DEL } \\
\text { PROCESO DE } \\
\text { ENSEÑANZA Y } \\
\text { APRENDIZAJE }\end{array}$} & \multirow{5}{*}{$\begin{array}{l}\text { Influencia de los } \\
\text { métodos de la Escuela } \\
\text { Nueva }\end{array}$} & Método Montessori \\
\hline & & Método Decroly \\
\hline & & Método de Proyectos (Dewey) \\
\hline & & Método Cousinet \\
\hline & & Método Freinet \\
\hline & \multicolumn{2}{|l|}{ Influencia de la ILE } \\
\hline & \multicolumn{2}{|c|}{ Influencia de la Escuela Moderna de Ferrer i Guardia } \\
\hline & \multicolumn{2}{|c|}{ Adecuación de la educación e individualización del aprendizaje } \\
\hline & \multicolumn{2}{|l|}{ Educación integral } \\
\hline & \multicolumn{2}{|l|}{ Educación activa } \\
\hline & \multicolumn{2}{|c|}{$\begin{array}{l}\text { El valor educativo de las excursiones escolares, de los juegos y de los } \\
\text { trabajos manuales }\end{array}$} \\
\hline & \multicolumn{2}{|c|}{ Relación entre la teoría y la práctica } \\
\hline & \multicolumn{2}{|c|}{ Cooperación y colaboración } \\
\hline & \multicolumn{2}{|c|}{ Aprendizajes funcionales y contextualizados } \\
\hline & \multicolumn{2}{|c|}{ Relación con la naturaleza y educación en el arte y en la belleza } \\
\hline & \multirow{2}{*}{ Educación para la paz } & Igualdad de género y coeducación \\
\hline & & Educación en otros valores positivos \\
\hline & \multicolumn{2}{|c|}{$\begin{array}{l}\text { El docente como guía que orienta el aprendizaje del alumnado y favorece } \\
\text { su motivación }\end{array}$} \\
\hline
\end{tabular}

Fuente: elaboración propia.

\section{El final de Escuelas de España}

Coincidiendo con el estallido de la Guerra Civil Española (17 de julio de 1936) se truncan gran parte de las iniciativas renovadoras pedagógicas que habían aflorado durante el primer tercio del siglo. Es en este momento cuando Escuelas de España llega a su fin, con la publicación de su último número en julio de 1936. Según Checa (2002), desde entonces "se impondrá [...] una visión única, católico-autoritaria" (p. 30), que no solo supuso la depuración de un tercio de los docentes españoles de la enseñanza, sino que conllevaría un paulatino decaimiento de las revistas pedagógicas existentes hasta este momento, llegando a suprimirse más de 50 publicaciones educativas.

\section{Consideraciones finales}

Escuelas de España es una revista con una gran riqueza pedagógica. Sus páginas evidencian cómo en diferentes lugares de España se sucedieron experiencias que contribuyeron a renovar y mejorar la educación y las escuelas de la época, de la mano de personalidades importantes, algunas señaladas a lo largo de este artículo. Su contenido, la 
trayectoria vital y profesional de sus redactores, el contexto en el que se desarrolló y el posterior intento de exterminio que sufrió la revista, evidencia su conexión, no solo con el movimiento internacional de la Escuela Nueva, sino con la ILE, cuya pretensión fue la de llevar a cabo una reforma pedagógica que desembocase en la regeneración de España. Todo esto hace que sea un elemento fundamental en el conocimiento del pasado educativo y escolar, así como un abanico de oportunidades para valorar el alcance de la renovación pedagógica proyectada, además de una oportunidad de reconocimiento y recuerdo de los maestros y las maestras que lucharon para llevar una educación de calidad a todos los rincones del país. En este sentido, son necesarias iniciativas encaminadas a la recuperación de la revista, entendiendo que es parte del patrimonio histórico-educativo que puede ayudarnos a conocer la realidad escolar del primer tercio del siglo XX en España.

\section{Referencias}

AGUILERA, María Yolanda. Un caso-estudio sobre las revistas educativas: La Revista Educación (género y producción de conocimiento). Revista Complutense de Educación, v. 16, n. 2, p. 387-414, 2015.

ALFARO, Ignacio; CARPINTERO, Helio. La psicología en la educación. Un análisis a través de la "Revista de Pedagogía" (1922-1936). Revista de Historia de la Psicología, v. 4, n. 3, p. 197-223, 1983.

ALONSO, Roberto. Concepción Sáinz-Amor: burgalesa, maestra y pionera. Brotes, n. 6 , p. 13-16, 2009.

ANDRÉS, Pablo de; ZUFRIATEGUI, Ainhoa; ESTEBARANZ, Aku. La hora de la aldea. Navarra: Gráficas Castuera, 2015.

ANDRÉS, Soledad de; MORA, José Luis. De ley y de corazón. Historia epistolar de una amistad. María Zambrano Alarcón - Pablo de Andrés Cobos - Cartas (1957-1976). Madrid: Universidad Autónoma de Madrid, 2011.

ARITMENDI, Ángel Luis. Lorenzo Luzuriaga y la Revista de Pedagogía, crónica de un proyecto educativo truncado (1922-1936). Boletín de la Institución Libre de Enseñanza, n. 73, p. 87-102, 2009.

BALLARÍN, Pilar. ¿Una profesión feminizada? Cuadernos de Pedagogía, n. 469, p. $35-$ 37, 2016.

BASTOS, Maria Helena Camara. A imprensa de educação e de ensino: repertórios analíticos. O exemplo da França. Revista Brasileira de Educação, v. 12, n. 34, p. 166168, 2007.

A imprensa de educação e ensino: observatório da formação docente e discente (Brasil, 1950-1980). Archivos de Ciencias de la Educación, n. 10, p. 1-14, 2016, Traducción propia.

BLAT, Amparo. Herminio Almendros lbáñez: Época, vida y obra. Añil: Cuadernos de Castilla-La Mancha, n. 16, p. 63-66, 1998.

CACHAZO, Alexia. Prensa pedagógica en Cataluña (1820-1939). 2015. Tesis (Doctorado en Educación) - Programa de Perspectiva Histórica, Comparada y Política de la Educación, Universidad de Salamanca/Usal, Salamanca, 2015.

CAMPBELL, Louisa. Educação na primeira república: alfabetização e cultura escolar na Revista de Ensino (1902-1910). História da Educação, v. 17, n. 41, p. 177-194, 2013.

CASPARD-KARYDIS, Pénélope. La Presse d'éducation et d'enseignement 1941-1990. 
Répertoire analytique. París: Institut National de Recherche Pédagogique, 2000.

CHECA, Antonio. Historia de la prensa pedagógica en España. Sevilla: Universidad de Sevilla, 2002.

DIEGO, Carmen. Luis Huerta. Maestro e higienista. Oviedo: KRK, 2006.

DÍEZ, Alejandro; POZO, María del Mar del; SEGURA, Manuel. La "Revista de Escuelas Normales": una publicación de regeneración normalista nacida en Guadalajara (19231936). Revista Interuniversitaria de Formación del Profesorado, n. 1, p. 9-30, 1988.

DUEÑAS, Carlos de; GRIMAU, Lola. De las sombras a la luz. La educación en Segovia (1900-1931). Madrid: Talasa, 2009.

ERMEL, Tatiane de Freitas. Cultura material, espaços e edifícios escolares na Revista de Pedagogía/Espanha: a circulação das ideias internacionais e o contexto espanhol (19221934). História da Educação, v. 21, n. 51, p. 297-316, 2017.

ESTEBAN, León; LÓPEZ, Ramón. La prensa pedagógica en su devenir histórico (antecedentes de la revista española de pedagogía). Revista Española de Pedagogía, v. 50, n. 192, p. 217-256, 1992.

ESTEBARANZ, Aku. Pablo de Andrés Cobos: maestro en La Granja. Segovia, 1 mar. 2016. Disponible en: <https://goo.gl//Xrglr>. Acceso en: 31 oct. 2017.

FERNÁNDEZ, Manuel. Mi paso por el semicentenario colegio "EI Peñascal". EI Adelantado de Segovia, s. f. Disponible em: <https://goo.gl/VxZWGz>. Acceso en: 31 oct. 2017.

GARCíA, Norberto. La recuperación de Norberto Hernanz Hernanz (Maestro segoviano, fundador de los Centros de Colaboración Pedagógica). El Adelantado de Segovia, 2008. Recuperado de: <http://www.eladelantado.com/opinionAmplia/5536/opinion>.

GÓMEZ, Alberto Luis; ROMERO, Jesús. Escuela para todos: conocimiento académico y geografía escolar en España (1830-1963). Santander: Universidad de Cantabria, 2007.

GONZÁLEZ, Etelvino. El pedagogo Luis Álvarez Santullano. Cuadernos de la Cátedra Miguel de Unamuno, v. 1, n. 48, p. 119-175, 2010.

HERNÁNDEZ, José María. Prensa pedagógica y patrimonio histórico educativo. Salamanca: Universidad de Salamanca, 2013.

HERNANZ, Norberto. Memorias Norberto Hernanz y Hernanz. Manuscrito inédito, s. f. HERRERO, Clemente. La geografía en la Revista de Escuelas Normales. Estudios Geográficos, v. 57, n. 222, p. 31-65, 1996.

. Índice de la "Revista de Escuelas Normales". Revista de Educación de la Universidad de Granada, n. 10, p. 287-309, 1997.

LÓPEZ, José Damián; DELGADO, María Ángeles. La enseñanza de las ciencias escolares en la Revista de Pedagogía (1922-1936). Educació i Història: Revista d'Història de l'Educació, n. 24, p. 69-101, 2014.

LOZANO, Claudio. La educación republicana 1931-1939. Barcelona: Universitat de Barcelona, 1980.

MARÍN, Teresa. La renovación pedagógica en España (1907-1936). Los pensionados en pedagogía por la Junta para la Ampliación de Estudios. Madrid: Artes Gráficas, 1990.

MARTÍNEZ, Eulalia. El Boletín de la Institución Libre de Enseñanza B.I.L.E. Contextos Educativos, n. 2, p. 71-78, 1999.

MELLADO, Judith. María Baldó Massanet, pedagoga, activista y feminista, 11 feb. 
2017. Disponible en: <https://goo.gl/Si9gt4>. Acceso en: 27 nov. 2017.

MERINO, Juan Carlos. El Bile en el siglo XXI. Historia Actual Online, n. 27, p. 189-202, 2012.

MONTES, Soledad; BEAS, Miguel. Presencia de Pestalozzi en La Escuela Moderna (1891-1934). Revista Complutense de Educación, v. 9, n. 1, p. 165-176, 1998.

MORA, José Luis; HERMIDA, Fernando. En torno a Ortega y Gasset, Machado y Zubiri. Epistolario: Norberto Hernanz - Pablo de A. Cobos (1934-1971). Revista de Hispanismo Filosófico, n. 16, p. 95-143, 2011.

ORTEGA, Fátima. La presencia de Ovide Decroly en el Boletín y en la Revista de Escuelas Normales (1922-1936). Bordón - Revista de Pedagogía, v. 66, n. 3, p. 121-131, 2014.

ORTIZ, Rosa. La renovación pedagógica desde la mirada de la revista Escuelas de España (1929-1931). 2017. Trabajo de Fin de Máster (Máster de investigación en Ciencias Sociales - Educación, comunicación audiovisual, economía y empresa) Universidad de Valladolid/UVA, Segovia. Recuperado de: <http://uvadoc.uva.es/bitstream/ 10324/24324/1/TFM-B.109.pdf>.

PALOMERO, José Emilio. La Revista Interuniversitaria de Formación del Profesorado. Una larga trayectoria, enraizada en el primer tercio del siglo XX. Revista Interuniversitaria de Formación del Profesorado, v. 78, n. 23, 3, p. 57-69, 2013. PETCHAK, Claudia María; MOURA, María Isabel. Imprensa periódica e a centralidade da educação no início do século XX. Imagens da Educação, v. 3, n. 1, p. 11-18, 2013.

RODRIGUES, Elaine. A imprensa pedagógica como fonte, tema e objeto para a história da educação. In: JUVENAL, Célio; PEREIRA, José Joaquim; HERMENEGILDO, Luiz (Orgs.). Fontes e métodos em história da educação. Dourados: Universidade Federal de Grande Dourados, 2010. p. 311-327.

SÁNCHEZ, Elena. Las Maestras de la República. Madrid: Catarata, 2012.

TIANA, Alejandro. La investigación histórico-educativa actual. Enfoques y métodos. Madrid: UNED, 1988.

VALLES, José Manuel. Vida intelectual en Segovia a comienzos del siglo XX. La Universidad Popular. En: Educación y cultura en Segovia, 1910-1931. XXXI Curso de Historia de Segovia. Segovia: Real Academia de Historia y Arte de San Quirce, 2010. p. 39-56.

VILLEGAS, Manuel; IBARZ, Virgili. La Revista de Psicología i Pedagogía de Barcelona (1933-1937). Revista de Historia de la Psicología, v. 14, n. 1, p. 1-13, 1993.

VIÑAO, Antonio. La modernización pedagógica española a través de la "Revista de Pedagogía" (1922-1936). Anales de Pedagogía, n. 12-13, p. 7-45, 1994-1995.

ROSA ORTIZ DE SANTOS es graduada en Magisterio de Educación Primaria y titulada en el Máster de investigación en Ciencias Sociales - Educación, comunicación audiovisual, economía y empresa. Contratada predoctoral en el Departamento de Pedagogía de la Facultad de Educación de Segovia (Universidad de Valladolid, España).

Dirección: Plaza de la Universidad, 1, 40005, Segovia, España.

E-mail: rosa.ortiz@uva.es 
LUIS TORREGO EGIDO es profesor titular en el Departamento de Pedagogía de la Universidad de Valladolid (España). Doctor en Filosofía y Ciencias de la Educación por la Uned. Coordinador de la Comisión Académica del Programa de Doctorado en Investigación Transdisciplinar en Educación de la Universidad de Valladolid.

Dirección: Plaza de la Universidad, 1, 40005, Segovia, España.

E-mail: Itorrego@pdg.uva.es

Recebido em 30 de novembro de 2017.

Aceito em 30 de julho de 2018. 